\title{
The Riskiest Job in Medicine: Transplant Surgeons and Organ Procurement Travel
}

\section{J. Englesbe* and R. M. Merion}

\author{
Department of Surgery, University of Michigan, Ann \\ Arbor, MI \\ * Corresponding author: Michael J. Englesbe, \\ englesbe@med.umich.edu \\ Funding source: None.
}

\begin{abstract}
Transplant surgeons are exposed to workplace risk due to the urgent nature of travel related to organ procurement. A retrospective cohort study was completed using data from the Scientific Registry of Transplant Recipients and the National Transportation Safety Board. A web-based survey was administered to members of the American Society of Transplant Surgeons. The survey response rate was $38 \%(281 / 747)$. Involvement in $\geq 1$ procurement-related travel accident was reported by $15 \%$ of respondents; surgeons reported 61 accidents and 11 fatalities. Air travel was used in $26 \%$ of procurements and was involved in $56 \%$ of accidents. The risk of fatality while traveling on an organ procurement flight was estimated to be $\mathbf{1 0 0 0}$ times higher than scheduled commercial flight. Involvement in a 'near miss accident' was reported by $80.8 \%$. Only $16 \%$ of respondents reported feeling 'very safe' while traveling. Procurement of organs by the geographically closest transplant center would have reduced the need for air travel (>100 nautical miles) for lung, heart, liver and pancreas procurement by $35 \%, 43 \%, 31 \%$ and $49 \%$, respectively (p $<0.0001)$. These reductions were observed in each Organ Procurement and Transplantation Network region. Though these data have important limitations, they suggest that organ procurement travel is associated with significant risk. Improvements in organ procurement travel are needed.
\end{abstract}

Key words: Donor management, donor risk, population studies, mortality

Received 12 March 2009, revised 05 June 2009 and accepted for publication 08 June 2009

\section{Introduction}

Being a doctor in the United States is a safe job. The overall physician workplace fatality rate is less than 0.5 per 100000 employed and between 1.0 and 4.0 per 100000 among surgeons (1). These rates compare favorably to the average workplace fatality rate of 4.0 per 100000 .
It is plausible, however, that transplant surgeons have a markedly higher risk of workplace mortality because of the urgent nature of travel related to deceased donor organ procurement. Organ procurement efforts require a team of surgeons, perfusionists and, in many cases, pilots. In contrast to the low rates of workplace-related death among medical professionals, commercial pilots have a reported fatality rate of 66.9 per 100 000, which is among the highest workplace fatality rates in the United States (1).

While management of deceased organ donors and care of transplant recipients have benefited from remarkable advances, organ procurement travel practices have remained largely unchanged and virtually unstudied. Similarly, despite broad expansion of aeromedical transport, there is concern that such flights are becoming less safe $(2,3)$. Given this background, clinicians who travel on organ procurement trips are called upon to face risks not borne by the vast majority of physicians. Yet they focus upon the urgency and perceived importance of the task at hand and may tend to take their own personal safety for granted. Tragedy has been too common, with at least seven documented fatal crashes and 27 documented fatalities in recent history (Table 1).

Every transplant surgeon has a story of his or her worst organ procurement trip, and the bravado and daring of these potentially perilous situations have often been worn as badges of honor. The recent occurrence of an organ procurement travel-related tragedy, involving the loss of a six-person transplant team from the University of Michigan, has again focused attention on the inherent risks. To address this issue, we surveyed the members of the American Society of Transplant Surgeons (ASTS) and also analyzed data from the Scientific Registry of Transplant Recipients (SRTR) to learn more about organ procurement travel practices in the United States. Our data must be considered within their limitations, but suggest that there are unnecessary risks and inefficiencies in the current systems used by surgeons to procure organs for transplantation and that many potential opportunities exist for improvement.

\section{Methods}

\section{Survey of transplant surgeons}

We conducted a 50-item, web-based survey, jointly sponsored by the ASTS and the University of Michigan (see the Appendix). The aim was to gather 
Table 1: Publicly reported fatal organ procurement accidents

\begin{tabular}{|c|c|c|c|c|}
\hline Transplant center & Year & Mode of travel & Location & $\begin{array}{l}\text { Number of } \\
\text { fatalities }\end{array}$ \\
\hline University of New Mexico & 1990 & Fixed wing aircraft & New Mexico, USA & 2 \\
\hline Ruhr University Bochum & 1994 & Helicopter & Bad Oeynhausen, Germany & 1 \\
\hline $\begin{array}{l}\text { Santa Casa de Porto Alegre, } \\
\text { Rio Grande do Sul }\end{array}$ & 1997 & Fixed wing aircraft & Florianopolis, Brazil & $\begin{array}{l}7 \\
7\end{array}$ \\
\hline California Transplant Donor Network & 2000 & Fixed wing aircraft & California, USA & 1 \\
\hline Brotzu Hospital of Cagliari & 2004 & Fixed wing aircraft & Sardinia, Italy & 6 \\
\hline University Hospital of Besançon & 2006 & Fixed wing aircraft & Besancon, France & 4 \\
\hline University of Michigan & 2007 & Fixed wing aircraft & Wisconsin, USA & 6 \\
\hline
\end{tabular}

data on current organ procurement travel practices and detail travel-related accidents in the United States. The survey was tested by 10 surgeons from three sites (University of Michigan, University of Alabama Birmingham and University of Massachusetts) and appropriate edits were made.

The Survey Research Center, Institute for Social Research at the University of Michigan administered the survey. The survey itself was managed using DatStat Illume 4.5 (DatStat Inc., Seattle, WA). An e-mail was sent to all members of the ASTS announcing the survey. Data collection for the web-based survey was opened on October 16, 2007 and was closed on November 9, 2007. Four separate reminder e-mails were sent to potential respondents. Data were extracted from every survey from which the respondent logged into the survey Web site. The final data set excluded 14 cases where no questions were answered and 28 cases of respondents who did not practice in the United States.

Data analyses were performed using SAS software, version 9.1 (SAS Institute, Cary, NC).

\section{Analysis of national organ procurement travel distance data}

We calculated the average organ procurement travel distance in the United States for heart, lung, liver and pancreas transplants. National data on all transplant candidates, recipients and donors are collected by the Organ Procurement and Transplantation Network (OPTN) and analyzed by the SRTR under a contract administered by the Health Resources and Services Administration, United States Department of Health and Human Services. We limited our analysis to procurement of deceased donor organs used for transplant in 2006. For each transplant, we used global positioning system coordinates to identify the specific planetary location for the donor hospital, the recipient transplant center and the transplant center (that performed at least one of that type of organ transplant in 2006) that was closest to the donor hospital. We then calculated the distance between the donor hospital and the recipient transplant center and the distance between the donor hospital and the closest transplant center for each transplant. Kidney procurements were not included in this analysis, because they are often done by surgeons not affiliated with a transplant center. As a result, we were unable to calculate travel distances for kidney procurements.

We selected 100 nautical miles as a threshold distance above which air travel would likely be utilized and below which ground transportation would be preferred. For each OPTN region and organ type, we calculated the proportions of transplants where the distance from the actual or closest transplant center to the donor hospital exceeded 100 nautical miles.

\section{Estimating the fatality risk of organ procurement travel}

Using data from the OPTN, we calculated the average number of heart, lung, liver and pancreas transplants completed between 1990 and 2007 (4) We then estimated the number of procurement trips that travel by air per year. This estimate was based on our analysis of data from the survey by the SRTR, in which $18 \%$ of procurements required no travel since the donor operation occurred in the same hospital as the recipient operation. In addition, we assumed that all procurement trips greater than 100 nautical miles utilized either a helicopter for a fixed wing aircraft for travel. We estimated the average airspeed to be 244 miles/h. Average airspeed was calculated as a weighted mean of estimated fixed wing (300 miles/h) and helicopter (120 miles/h) airspeeds. Data from the ASTS survey reported that $19 \%$ of air travel utilized helicopters. We then calculated the total number of flight hours over the 18-year observation period. There have been nine known fatalities in the United States between 1990 and 2007 during air travel for organ procurement. The number of fatalities was determined by both the survey and a query of data from the National Transportation Safety Board (NTSB) accident database. In addition, the number of accidents reported to the Federal Aviation Administration (FAA) between 1990 and 2007 were determined by query of the NTSB database. The fatality rate was reported as fatalities per million flight hours, which is the standard format of the NTSB (5)

This study was approved by the Institutional Review Board at the University of Michigan.

\section{Results}

\section{Organ procurement travel practices}

The survey response rate was 38\% (281/747). The respondents represented all 11 OPTN regions and 51 of 58 organ procurement organizations (OPOs) in the United States. Cardiothoracic transplantation was practiced by $8.2 \%(\mathrm{~N}=$ 23) of respondents and the remainder practiced abdominal transplantation. Respondents reported that they had been in transplant surgery practice for a median of 13.2 years (interquartile range [IQR] 8.1, 18.8), had performed 10.0 (IQR 2.0, 18.8) procurements within the last year and had generated $4.2 \%$ (IQR $0,10.0)$ of their income from procurement activities. The plane crash involving the University of Michigan transplant team that occurred 4 months prior to the survey was cited by 105 respondents $(41.7 \%)$ as having affected how they answered the survey questions.

The modes of travel used by surgeons and their teams are detailed in Figure 1A. Overall, air travel (fixed wing aircraft and helicopter) was reported for $26 \%$ of all-organ procurement trips. Thoracic surgeons were 2.1 times more likely to fly and 3.0 times more likely to use a helicopter ( $p<0.05$ for both). There was significant regional variation in modes of travel, with surgeons from less densely 


\section{Englesbe and Merion}

A

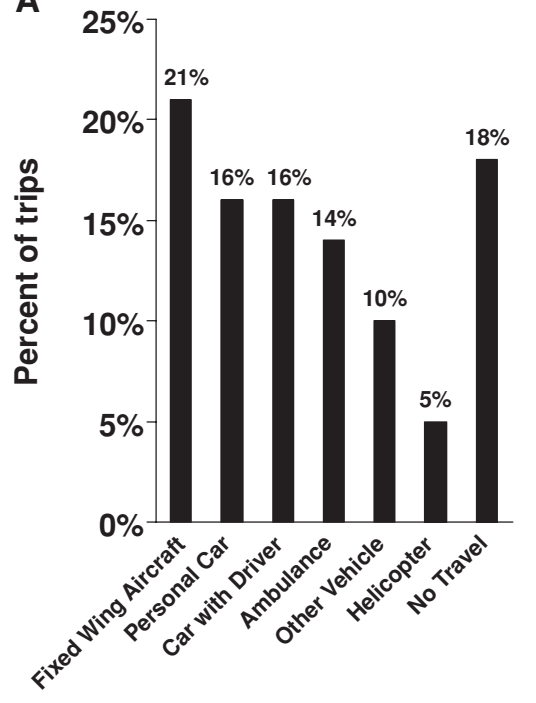

B

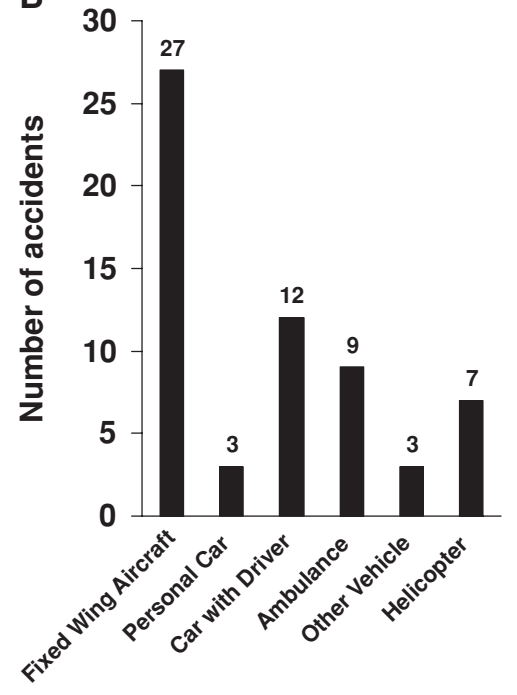

Figure 1: Modes of travel and accidents during organ procurement travel. Panel A depicts modes of travel used to procure organs by transplant surgeons. Donors at the home institution, which accounted for $18 \%$ of procurements, were considered to require no travel. Panel B depicts the number of respondent-reported accidents by mode of travel. The total number of reported accidents was 61 populated areas being more likely to utilize aircraft (Figure 2A). Surgeons were most likely to be accompanied by an organ transplant perfusionist, surgical resident or fellow and least likely to travel with another attending surgeon (Table 2).

\section{Organ procurement travel risk}

Overall, $15 \%$ of respondents (42 surgeons) stated that they had been involved in at least one travel-related accident and $56 \%$ of reported accidents occurred during air travel (Figure 1B). Overall, surgeons reported personal involvement in 61 accidents. Importantly, surgeon reports of accident involvement differed markedly from FAA-documented accidents. More specifically, upon query of NTSB data, there were only seven accidents involving organ procurement travel reported to the FAA between 1990 and 2007 (Table 3).

One-third of respondents stated that personnel at the transplant center at which they currently work had experienced an accident while procuring donor organs; a total of 122 accidents and 68 injuries were reported. Further, surgeons reported being in a 'dangerous situation without an accident' or 'having a near miss' a median of 2.3 times (IQR 0.2,4.7) over the course of their career. Less than one in five $(19.2 \%, N=54)$ reported that they had never been involved in a 'near miss'. Three of the seven fatal accidents shown in Table 1 were recounted by respondents, with a total of 11 deaths. One accident resulted in six fatalities (United States, 2007), one accident had four deaths (France, 2006) and one was associated with a single fatality (United States, 2000).

Only $16 \%$ of respondents reported feeling 'very safe' while traveling (Table 2). The highest reported levels of concern, reported as medians based on a five-point Lickert scale $(1=$ never, $2=$ rarely, $3=$ sometimes, $4=$ often and 5
= always) included: weather (median 3, IOR 3, 4), spouse worrying about them (median 3, IQR 2, 4), driver fatigue (median 2, IQR 2, 3), mechanical failure (median 2, IQR 1, 3 ), pilot error (median 2, IQR 1, 2) and pilot fatigue (median 1, IQR 1, 2). Respondents reported that they had life insurance policies covering organ procurement accidental death funded by: the hospital (32.6\%), the OPO (12.6\%) or themselves (25.4\%). Interestingly, $29.3 \%$ of respondents reported that they were unsure if they had life insurance that would cover a fatal accident while traveling for organ procurement.

Based on data from the OPTN, and our analysis of data from the SRTR, the NTSB and our survey, we estimated the risk of fatality while traveling by air to procure organs for transplant in the United States between 1990 and 2007 to be 54.8 fatalities per million flight hours (Table 4).

\section{Organ procurement travel inefficiency}

Among liver transplant surgeons, $85.5 \%$ of respondents reported being willing to have surgeons from other liver transplant centers procure livers for them, but another team actually procured livers only $14.8 \%$ of the time. A majority of heart $(68.7 \%)$ and lung $(61.1 \%)$ transplant surgeons reported that they were willing to have surgeons from another center procure organs for them. However, these respondents reported that another heart or lung team actually procured organs for them only $1.1 \%$ and $5.6 \%$ of the time, respectively. The majority agreed that existence of a mechanism to facilitate better communication would increase the likelihood of allowing another transplant center to procure organs $168.0 \%, 78.6 \%$ and $73.3 \%$ of liver, heart and lung transplant surgeons, respectively).

Using SRTR data for organ procurements in 2006 (heart, $N=2142$; lung, $N=1346$; liver, $N=5831$ ), we determined 


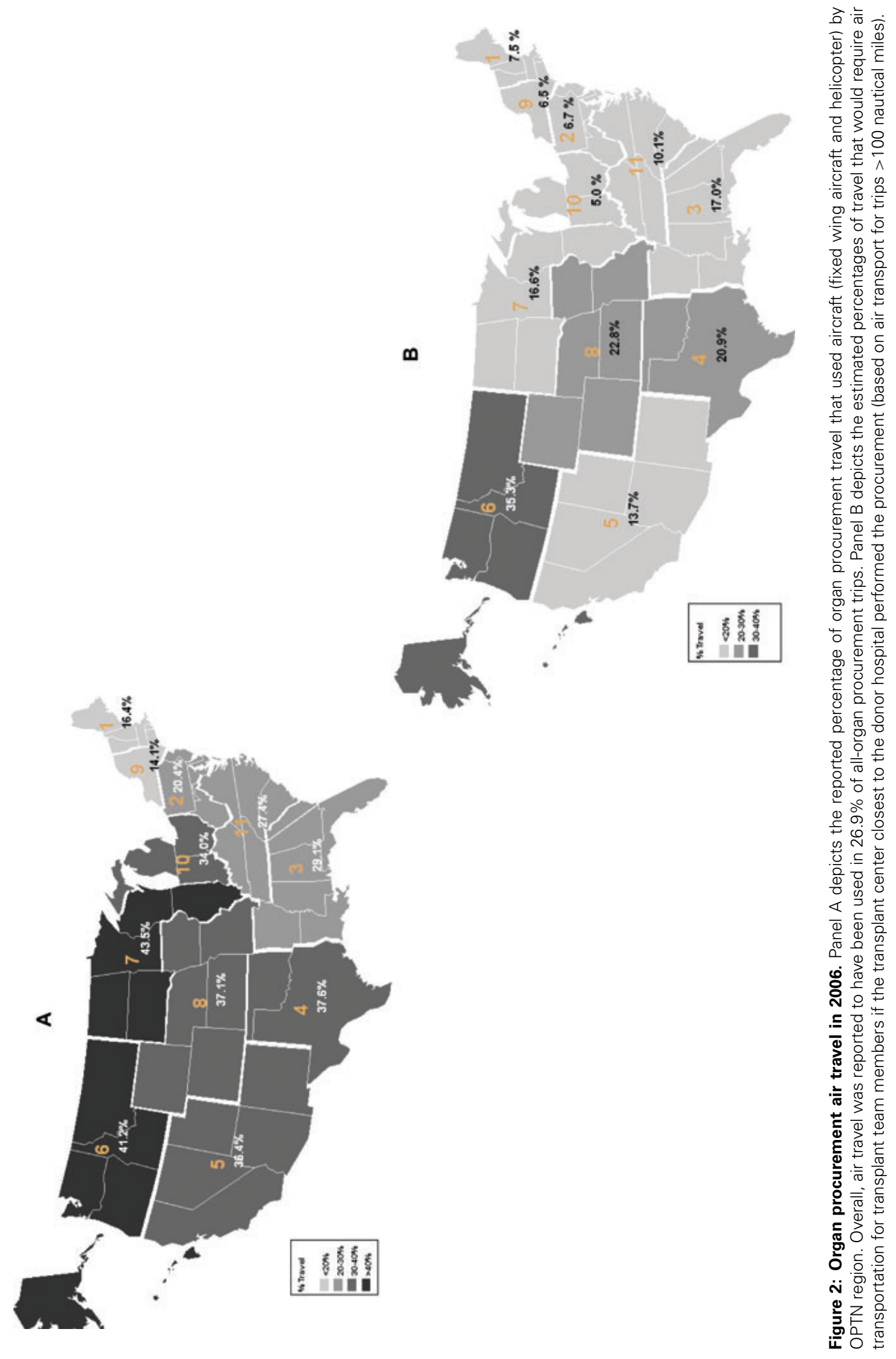




\section{Englesbe and Merion}

Table 2: Responses to selected survey questions

\begin{tabular}{lr}
\hline & Percent \\
\hline Personnel accompanying transplant surgeon & \\
Perfusionist & \\
Never & 12.0 \\
Rarely & 2.0 \\
Sometimes & 8.8 \\
Often & 16.3 \\
Always & 61.0 \\
Surgical resident & \\
Never & 5.3 \\
Rarely & 13.4 \\
Sometimes & 29.7 \\
Often & 35.8 \\
Always & 15.9 \\
Surgical fellow & \\
Never & 28.2 \\
Rarely & 6.9 \\
Sometimes & 9.4 \\
Often & 32.2 \\
Always & 23.3 \\
Medical student & \\
Never & 14.3 \\
Rarely & 29.0 \\
Sometimes & 36.3 \\
Often & 19.6 \\
Always & \\
Another attending & \\
Never & \\
Rarely & \\
Sometimes & \\
Often & \\
Always & \\
How safe do you feel on organ procurement travel? & \\
Very unsafe & \\
Unsafe & \\
Usually safe & 21.6 \\
Safe & \\
Very safe & \\
\hline
\end{tabular}

the distance between the donor hospital and the actual transplant center as well as the closest transplant center that performed at least one transplant of the organ of interest in 2006 (Figure 3A). For each organ evaluated, the average distance between the closest transplant center and the donor hospital was less than one-third the distance

Table 3: Accidents during air travel for organ procurement reported by the National Transportation Safety Board

\begin{tabular}{lll}
\hline Date & Record no. & \multicolumn{1}{c}{ Aircraft } \\
\hline $7 / 15 / 1990$ & CHI90LA172 & LEARJET \\
9/11/1990 & DEN90FA184 & MORAINE-SAULNIER \\
$5 / 21 / 1998$ & MIA98LA167 & NORTH AMERICAN \\
$5 / 23 / 1998$ & ATL98LA078 & LEARJET \\
$11 / 6 / 2000$ & LAX01FA032 & CESSNA \\
$7 / 1 / 2001$ & LAX01FA252 & Sikorsky (helicopter) \\
$6 / 4 / 2007$ & CHI07MA160 & CESSNA \\
\hline
\end{tabular}

Table 4: Estimating the risk of fatality while traveling by air to procure organs for transplant in the United States between 1990 and 2007

Mean number heart, lung, liver and 8918 transplants pancreas transplants per year (1990 to 2007) (4)

Number procurement trips traveling by air 3510 trips per year ${ }^{1}$

Average distance per procurement $\quad 636$ miles

Average airspeed ${ }^{2}$

Average trip duration (flight hours) 244 miles/h

$2.6 \mathrm{~h}$

Total flight hours over 18 year study period $164270 \mathrm{~h}$

Fatalities in the USA over the 18 year 9 people study period

Estimated fatality rate for organ procure air travel

Estimated fatality rate for scheduled commercial flights (5)

Estimated fatality rate for unscheduled commercial flights (5)

54.8 per million flight hours

0.055 per million flight hours

7.1 per million flight hours

${ }^{1}$ Based on the estimate that $18 \%$ of procurements require no travel (donor operation occurs in the same hospital as the recipient operation) and that aircraft were used for all trips $>100$ miles. These estimates were informed by analysis of data from the SRTR.

${ }^{2}$ Average airspeed calculated as a weighted mean of estimated fixed wing (300 miles/h) and helicopter (120 miles/h) airspeeds. Data from the ASTS survey reported that $19 \%$ of air travel utilized helicopters.

from the actual transplant center to the donor hospital ( $p<0.0001$ for each organ type).

In order to estimate the proportion of trips that would require air transport, we focused on procurements greater than 100 nautical miles. As shown in Figure 3B, if the closest transplant center had traveled to procure organs rather than the actual transplant center, there would have been a $32.9 \%$ overall reduction in the requirement for air travel by surgeons and their teams $(p=0.0001$ for each organ type). These findings were observed in every OPTN region for each organ type (Figures 2B and 3B).

\section{Discussion}

Imagine if an infectious disease specialist had to be driven to a pharmaceutical manufacturing plant in an ambulance to get antibiotics for a septic patient, or an orthopedic surgeon had to fly to a factory to buy an artificial joint for a patient who needed a hip replacement. Of course, it is difficult to envision these improbable scenarios, because the requisite materials can be ordered and paid for, shipped by conventional means to hospitals and stored for long periods of time. Transplant surgery is unique in medicine because of the need for highly skilled surgeons and other trained personnel to travel, on extremely short notice and often to very distant locations, in order to secure a deceased donor organ that is fragile, highly perishable and only transiently available. This resource, on which the 
A
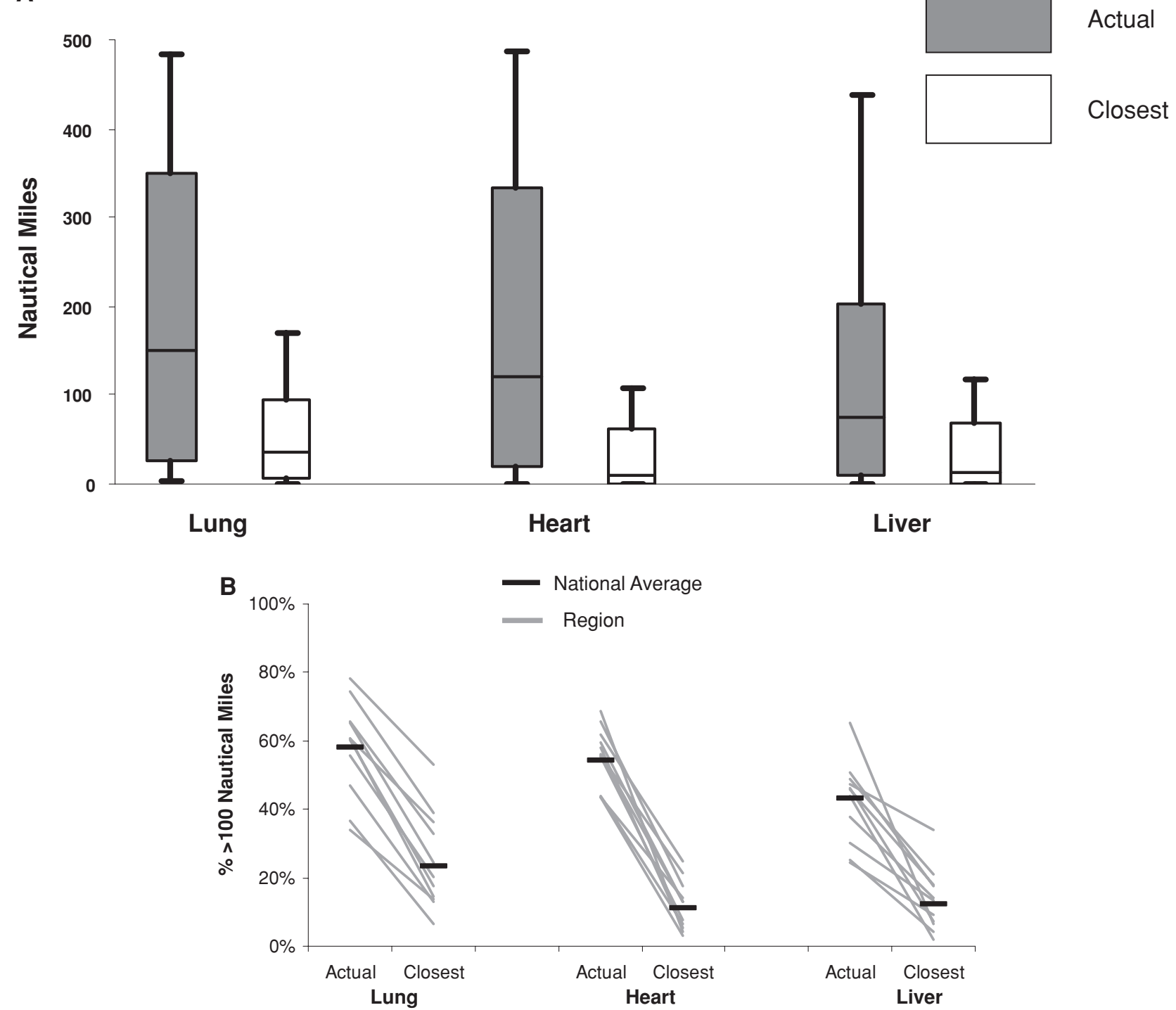

Figure 3: Organ procurement travel distances: actual vs. closest transplant center. Panel A depicts travel distances for heart, lung, liver and pancreas transplant procurements in 2006. Black box and whisker plots represent the distance between the donor hospital and the closest transplant center. Gray box and whisker plots represent the distance between the donor hospital and the actual transplant center. The horizontal bars represent the median; the boxes represent the IQR; the whiskers represent the 5th and 95th percentile values. Panel B depicts the estimated proportions of trips requiring air travel (based on air transport for trips $>100$ nautical miles) for the closest transplant center and the actual transplant center for organs procured in 2006.

patient's life and future health depends, cannot be requisitioned or purchased (6).

In this study, we have attempted to describe current organ procurement travel practices by transplant surgeons in the United States. It is critical that high level of fatality risk we calculated for organ procurement travel must be considered within the limitations of the available data. Nonetheless, we have documented deeply troubling occurrences of surgeon-reported dangerous situations, near misses, accidents and fatalities. Our survey demonstrated that transplant surgeons have deep-seated concerns about their safety and well-being and a willingness to reconsider organ procurement practices that are presently associated with unnecessary risk exposure. Taken together, the study provides much needed albeit incomplete insight into these issues and suggests a number of opportunities to improve both the safety and efficiency of organ procurement practice.

As described earlier, physicians generally have safe jobs when compared to other occupations. These limited data 


\section{Englesbe and Merion}

from the survey strongly suggest that transplant surgeons do not. It was striking that $15 \%$ of respondent transplant surgeons reported having been involved in an accident during organ procurement travel. The majority of reported accidents occurred during air travel and many were associated with serious injury or death. It is critical for policy makers to balance these observations with the fact that, over roughly same time period, there were only seven accidents reported to the FAA (Table 3). This observation suggests a potential distinction between the perception of 'accidents' by surgeons and actual accidents as defined by aviation authorities. The accidents that did occur involved small aircraft. Unlike large commercial airliners, only a small proportion of general aviation aircraft accidents result in fatalities (7). The 'near misses' that surgeons recounted posed potentially grave danger, involving emergency landings and mechanical failures. Adverse weather was often mentioned as a contributing factor. Despite their tacit acceptance of the risk inherent in organ procurement activities, transplant surgeons reported persistent concern about the safety of organ procurement travel.

There are opportunities to improve the safety of organ procurement travel. Examples include adoption of a more robust, integrated and formalized safety-based culture, with closer cooperation and communication between transportation providers (e.g. charter services and pilots, ambulance services and drivers), transplant surgical teams, OPOs and the OPTN. Similarly, transplant policy makers should assure that all transplant professionals who participate in the recovery of life-saving organs have both life and disability insurance that cover these duties. The transplant surgery community, in collaboration with other stakeholders, needs to take its share of responsibility, as they have the insight needed to balance the clinical urgency of travel with safety concerns.

Similarly, there are opportunities to improve the efficiency of organ procurement travel. Given that the typical deceased donor yields 3.5 organs and that the majority of surgeons in our survey expressed a strong preference to send their own team to the donor hospital, it is reasonable to conclude that multiple abdominal and thoracic teams are present at the majority of donor procurement procedures $(8,9)$. Our data suggest that most transplant surgeons would be willing to have local transplant surgeons procure organs, which could then be transported without transplant professionals traveling along with the organs. Efforts should be made to facilitate collaborative efforts between surgeons and transplant centers in order to increase the local procurement of organs. Importantly, local surgeons may not be willing to procure organs for other transplant centers. Policy efforts to increase the local procurement of organs must address this issue and provide sufficient incentives to stimulate the local procurement of organs. In addition, donors could potentially be transported to designated procurement facilities adjacent to transplant centers or transportation hubs. Policies promoting alterna- tive practices in organ procurement could markedly reduce the number of personnel that travel, the distance they travel and the need for air travel (Figure 3A). Such policies are particularly germane considering recent initiatives that may increase the regional sharing of organs, resulting in longer travel distances and presumably more travel by air.

The strength of our study is inclusion of survey responses from transplant surgeons across wide geographic areas and representing all major transplanted organs and every modality of organ procurement travel. In contrast, the findings of this work have several important limitations that need to be carefully considered. The survey response rate was 38\%, and although a higher response rate would have been preferred, our response rate compares favorably to other large surveys of transplant surgeons $(10,11)$. There is a strong likelihood of responder bias. Since the survey included a major focus on accidents related to organ procurement travel, surgeons who had been involved in travelrelated accidents or who had strongly held attitudes about organ procurement travel practices may have been more likely to respond. Also, 15\% of surgeons reported involvement in a perceived 'accident' and likely many of these accidents could have been classified as 'incidents' rather than 'accidents' under the official definition given in 49 CFR Part 830 (the Federal Aviation Regulations). As a result, we cannot accurately report 'accident' rates for organ procurement travel. In addition, it is likely that many 'near misses' were not reported or potentially not even noted by surgeons. Finally, survey was clearly identified as having originated at the University of Michigan, and 42\% of respondents indicated that the Michigan crash affected how they answered some of the survey questions. Overall, it is important to consider the flaws of these data and the limitations of these methods as policy initiatives are recommended.

Fatal accidents in the United States were confirmed via an analysis of data from the NTSB; thus, we have presumably accounted for all fatal accidents within the United States. We are aware of three organ procurement fatal accidents outside of the United States, though we cannot rule other additional international accidents not captured by our methods. Overall, a total of six accidents account for a total of 27 fatalities. For our estimation of the risk of fatality to transplant professionals while flying to procure organs, we only considered the nine fatalities that have occurred in the last 18 years within the United States. We calculated the risk of fatality while traveling by air to procure organs to be approximately 1000 times higher than the risk of fatality while traveling on a scheduled commercial airline flight in the United States. Importantly, our calculation of the risks of organ procurement air travel must be taken within the context of the limitations of available data. Interestingly, precise data regarding organ procurement travel is not available, even to aviation regulators. Thus, although our work provides unique insight into the risks of organ 
procurement travel, additional data on organ procurement travel would be needed to better document travel-related accidents and to guide future policies to optimize procurement activities. Nonetheless, we speculate this increased risk can be mitigated with best practices, including pilot training, improvements of aeromedical carrier safety culture and importantly separating travel decisions from any consideration of clinical urgency.

While many aspects of organ transplantation are highly regulated, subject to extensive oversight and reported to the public, organ procurement travel practices are not. Regulatory oversight and consistency of practice in organ procurement travel may improve efficiency, reduce risk and possibly improve transplant outcomes through adoption of best clinical, travel and insurance practices. This would potentially reduce cost and benefit transplant surgeons, insurance carriers and ultimately the transplant patients who receive these precious organs. In addition and importantly, any policy initiatives must carefully consider the inherent limitations of this work.

\section{Acknowledgments}

We gratefully acknowledge the contributions of Jennifer Sinibaldi, Institute for Social Research, Ann Arbor, MI; Elaine Berg, New York Organ Donor Network, New York, NY; Jeffrey D. Punch, MD, University of Michigan, Ann Arbor, Ml; Keith C. McCullough, Arbor Research Collaborative for Health, Ann Arbor, Ml; and David Kenny, Aircraft Owners and Pilots Association-Air Safety Foundation, Frederick, MD, for their assistance.

\section{References}

1. National census of fatal occupational injuries in 2005. Bureau of Labor Statistics, U.S. Department of Labor 2006; http://www.bls.gov/iif/oshcfoi1.htm. Accessed March 11, 2009.

2. Bledsoe BE, Smith MG. Medical helicopter accidents in the United States: A 10-year review. J Trauma 2004; 56: 1325-1328; discussion 1328-1329.

3. Levin A, Davis R. Surge in crashes scars air ambulance industry. USA Today 2005; http://www.usatoday.com/news/nation/200507-17-air-ambulance-crashes_x.htm. Accessed July 17, 2005.

4. The Organ Procurement and Transplantation Network-Data on transplant activity. 2008. www.optn.org. Accessed May 11, 2008.

5. National Transportation Safety Board-Annual Review of Aircraft Accident Data. Calendar year 2004 report. 2008. http://www.ntsb.gov/publictn/2008/ARC0801.pdf. Accessed April 11, 2008.

6. Pub L No. 98-507. National Organ Transplant Act, 42 USC §273.

7. Rostykus PS, Cummings P, Mueller BA. Risk factors for pilot fatalities in general aviation airplane crash landings. JAMA 1998; 280: 997-999.

8. Punch JD, Hayes DH, LaPorte FB, McBride V, Seely MS. Organ donation and utilization in the United States, 1996-2005. Am J Transplant 2007; 7(5 Pt 2): 1327-1338.

9. 2007 Annual Report of the U.S. Organ Procurement and Transplantation Network and the Scientific Registry for Transplant Recipients: Transplant Data 1997-2006: Department of Health and Human Services, Health Resources and Services Adminis- tration, Office of Special Programs, Division of Transplantation, Rockville, MD; United Network for Organ Sharing, Richmond, VA; Arbor Research Collaborative for Health, Ann Arbor, MI. 2008. www.ustransplant.org. Accessed March 11, 2009.

10. Charpentier KP, Mavanur A. Removing patients from the liver transplant wait list: A survey of US liver transplant programs. Liver Transpl 2008; 14: 303-307.

11. Oz MC, Kherani AR, Rowe A et al. How to improve organ donation: Results of the ISHLT/FACT poll. J Heart Lung Transplant 2003; 22: 389-410.

\section{Appendix}

Organ Procurement Travel Practices Survey

1) Please enter your login:

2) Please enter the name of your Transplant Program.

3) What UNOS Organ Procurement Region is your center in?

4) What OPO is your center in?

5) How many years have you been practicing transplant surgery?

6) Are you currently a transplant surgery fellow?

7) Please specify the organ(s) that you personally transplant.

Heart

Intestine

Kidney

Liver

Lung

Pancreas

None of the above

8) How many deceased donor organ procurement operations did you personally perform between July 1, 2006 and June $30,2007 ?$

9) Which of the following types of transportation were used in these deceased donor organ procurement operations?

Fixed wing aircraft helicopter

Your personal car (to drive to the donor hospital) Ambulance

Hired vehicle with a professional driver (such as taxis or hospital-owned vans)

Vehicle owned and operated by the OPO

Other (please specify)

'In-house' donors where no travel was required

10) For each of the types of transportation you specified, please indicate what percent of your procurement operations included each type. Consider each type of transportation separately. The sum of the percentages will likely exceed 100\%, unless you only took one type of transportation for each procurement operation. For example, if you took a fixed wing aircraft on all of the donors and an ambulance on all of the donors, each would be recorded as $100 \%$.

Fixed wing aircraft

Helicopter

Your personal car

Ambulance 


\section{Englesbe and Merion}

Hired vehicle with a professional driver

Vehicle owned and operated by the OPO

'In-house' donors where no travel was required.

11) When doing an organ recovery, who makes arrangements for travel?

Your Hospital/Transplant Center

Practice group

The OPO

Other (please specify)

12) When doing an organ recovery outside of your center for organs to be used by YOUR center, you are functioning as which of the following?

Staff/faculty member of your transplant center

Independent contractor

Employee of the OPO

Other (please specify)

13) When doing an organ recovery outside of your center for organs to be used by YOUR center, who provides your insurance coverage against a travel related accident?

The transplant center or hospital life and disability plan Personal life and disability plans

OPO life and disability plans

Unsure

14) When doing an organ recovery outside of your center for organs to be used by ANOTHER center, you are functioning as which of the following?

Staff/faculty member of your transplant center Independent contractor

Employee of the OPO

Other (please specify)

15) When doing an organ recovery outside of your center for organs to be used by ANOTHER center, who provides your insurance coverage against a travel related accident?

The transplant center or hospital life and disability plans Personal life and disability plans

OPO life and disability plans

Unsure

How much influence does each of the following have on the method of travel? Please answer with one of the options below for questions 16-21.

No Influence
A Little Influence

Moderate Influence

A Lot of Influence

Very High Influence

16) Donor surgeon preference?

17) Recipient surgeon preference?

18) Transplant center policy?

19) OPO policy?

20) Ischemia times?

21) Pilot/flight team?

22) What percent of the time do you use HEARTS for transplant that have been recovered by a surgeon who does not work for your transplant center?

23) Which of the following best describes your preference for using HEARTS that have been recovered by a surgeon who does not work for your transplant center?

I always want a member of my team procuring the heart.

I would prefer a member of my team procuring the heart when possible.

I would be fine with another surgeon, outside my team, performing the organ procurement.

24) Would a program supporting exceptional communication between the donor and recipient surgeon make you more likely to allow a surgeon not from your team to procure HEARTS for you?

25) Would a web-based program that enabled you to assess the organ real time prior, during and following procurement make you more likely to allow a surgeon not from your team to procure HEARTS for you?

26) Would a program supporting a donor surgeon report-card system make you more likely to allow a surgeon not from your team to procure HEARTS for you?

Questions 22 through 26 repeated for each organ the respondent indicated that they transplant.

When you travel for an organ procurement, how often are the following people traveling with you, besides yourself? Please answer with one of the options below for questions 27-31.

Never

Rarely

Sometimes

Often

Always

27) An attending surgeon? 
Donor Travel Risks

28) A surgical fellow?

29) A surgical resident?

30) A medical student?

31) An organ preservation or perfusion specialist?

32) Does your center have a policy that medical students are not allowed to travel by air?

33) Over the course of your career, have you ever PERSONALLY been involved in a transportation accident while traveling on an organ procurement?

34) Over the course of your career, has the CENTER YOU WORKMORKED AT ever been involved in a transportation accident while traveling on an organ procurement?

35) How many transportation accidents have you PERSONALLY been involved in while traveling on an organ procurement? Please specify the mode of travel in which this accident occurred.

Private car (driven by a professional driver)

Personal car (driven by the surgeon or team member)

Ambulance

Helicopter

Fixed wing aircraft

Other (please specify)

36) How many people were injured?

37) How many people died?

The above questions regarding personal involvement in an accident were repeated depending upon the number of accidents the respondent reported and for the number of accidents the respondent reported at the Center where they are currently employed.

38) Over the course of your career, approximately how many times has your organ procurement team encountered a dangerous situation while traveling, which did not result in an accident?

Please describe the most recent event.

39) When traveling for an organ procurement, how safe do you feel?
Very Unsafe

Unsafe

Usually Safe

Safe

Very Safe

40) Consider each of the following things you might worry about while traveling for a procurement. Please indicate how often you worry about each. Please answer with one of the options below for questions 41-46.

Never

Rarely

Sometimes

Often

Always

Not Applicable

41) Fatigue of the person driving the team?

42) Fatigue of the person flying the team?

43) Mechanical failure?

44) Operator failure?

45) Weather?

46) My spouse or significant other worrying about me.

47) Is there anything else that you might worry about while traveling for a procurement?

Please specify what else you might worry about while traveling on a procurement.

48) Has the plane crash and deaths of members of the University of Michigan organ procurement team affected how you answered some of the questions on this survey?

49) What percent of your annual income for 2007 will come from organ procurement operations?

50) Please note any additional comments you have on trave policies and practices for organ procurement. 